4D.004 DIRECTIONAL HEARING AWARENESS, A NEW WAY TO PREVENT ACCIDENTS FOR HELMET USERS

Felipe Morales*. Efem Acoustics, Pereira, Colombia

10.1136/injuryprev-2021-safety. 111

Background Road traffic injuries constitute a major public health with consequences on mortality and morbidity, according to WHO Globally Approximately 1.35 million people die each year as a result of traffic crashes, approximately $25 \%$ where motorcycle drivers

Methods Traditional helmets cover the ears and dramatically reduce hearing acuity, listening to danger can be as important as seeing it. For this, a helmet with a system that improves hearing acuity without affecting impact protection was designed, patented, prototyped and tested in a certified through calculations of measures of central tendency for frequency by analysis of variance (ANOVA) with a confidence interval of $95 \%,(p<0.05)$ For the study of laterality, a Binomial analysis was applied to verify the probability of error and success with a confidence level of 95\%.

Results the research showed that the use of our Hearing Helmet gave the user an average sound source localization of $90 \%$. For the full-face Helmet user, it presented an average of $67 \%$, it presented difference on the precision of localization source between both helmets

Conclusion The awareness in real time of the surroundings that Hearing Helmet returns to motorcycle drivers, allows to have agile responses in the situations of the road. Implementing this solution will Increase road safety and help reduce worldwide accidents.

Learning Outcomes By improving hearing acuity while wearing a Hearing Helmet, accidents can be reduced and save lives. Hearing Helmet is a solution for road safety on populated urban areas. That is SDG 3.6.1 and our mission too.

\section{D.005 UNSAFE BICYCLING BEHAVIOR IN CHANGSHA, CHINA: A VIDEO-BASED OBSERVATIONAL STUDY}

\begin{abstract}
1,2Yuyan Gao, ${ }^{3}$ David Schwebel, ${ }^{4}$ Lingling Zhang, 'Wangxin Xiao*, 'Guoging Hu. ${ }^{1}$ Department of Epidemiology and Health Statistics, Xiangya School of Public Health, Central South University, Changsha, Hunan, China; ${ }^{2}$ Dongcheng Center for Disease Control and Prevention, Beijing, China; ${ }^{3}$ Department of Psychology, University of Alabama at Birmingham, Birmingham, USA; ${ }^{4}$ Department of Nursing. College of Nursing and Health Sciences, University of Massachusetts Boston, Boston, USA
\end{abstract}

\subsection{6/injuryprev-2021-safety.112}

Background The increased risky use of bicycles in China lead to growing bicycling injuries. We report the incidence of unsafe bicycling behavior and examine incidence differences across type of riders and areas cycled in.

Methods A video-based observational study was conducted to estimate the incidence of five unsafe bicycling behaviors in Changsha, China: not wearing helmets (A), violating traffic lights (B), riding in a direction opposite (C), holding the handlebar with one or no hands (D), and riding in a non-bicycle lane (E). Chi-square tests examined differences in unsafe cycling behavior incidence between shared versus personal bicyclists and across the area cycled in (commercial, university, office, or leisure areas). Logistic regressions quantified the association between unsafe cycling behaviors and both type of riders and areas cycled in.

Results The incidences of A, B, C, D, E were 99.28\% (95\% CI: $99.14 \%-99.41 \%), \quad 19.57 \% \quad(95 \%$ CI: $18.50 \%-20.64 \%)$, 13.73\% (95\% CI: 13.19\%-14.27\%), 2.57\% (95\% CI: $2.32 \%-$ 2.82\%), and 64.06\% (95\% CI: 63.16\%-64.96\%), respectively. Compared to personal bicycle riders, shared bicyclists had higher incidence rates of $\mathrm{A}$ and $\mathrm{C}(\mathrm{AOR}=18.97,2.08)$ but lower incidence of $\mathrm{B}(\mathrm{AOR}=0.63)(\mathrm{p}<0.05)$. Across the types of cycling areas, the university, commercial and office area had the highest incidence of $\mathrm{A}, \mathrm{C}$ and $\mathrm{B}$, respectively $(\mathrm{p}<0.05)$.

Conclusion Cyclists using shared and personal bikes were observed behaving in unsafe manners frequently, especially rarely wore helmets.

Learning Outcomes It highlights the unsafe cycling behaviors in urban China, and the high incidence of not wearing helmets are needed to be interpreted.

\section{E - Rural, March 24, 2021}

\section{E.001 LEARNINGS OF FARMER BEHAVIOUR FROM THE VICTORIAN QUAD BIKE REBATE SCHEME}

${ }^{1}$ Jimmy Twin*, 'Samantha Barker, '2Lauren Day, 'Janine McMillan, ${ }^{3} J a c q u i e$ Cotton, ${ }^{3}$ Jessie Adams, ${ }^{1}$ Frances Taylor, ${ }^{2}$ Andrea de Silva. 'Monash University - ISCRR, Melbourne, Australia; ${ }^{2}$ WorkSafe Victoria, Geelong, Australia; ${ }^{3}$ National Centre for Farmer Health, Hamilton, Australia

\subsection{6/injuryprev-2021-safety. 113}

Background Quad bikes are a cause of death and serious injury in Australian rural workplaces, with many incidents associated with rollovers. Since 2016, the Victorian Government has funded a rebate scheme for the farming sector to recover costs associated with fitting of a suitably designed and engineered operator protective device (OPD), or for the purchase of an approved side-by-side vehicle with rollover protection and fitted seat belts. The rebate scheme was integrated with an extensive television, print and social media campaign.

Methods An evaluation of the rebate process was carried out by the Institute for Safety, Compensation and Recovery Research (ISCRR). This included an electronic survey completed by 334 farmers and qualitative interviews $(n=51)$ to gain insights into farmer decision-making behaviour.

Results This evaluation identified key factors in determining farmer decision-making in relation to the rebate scheme and were mapped against a health belief model. Barriers to uptake included the initial financial outlay (in particular with side-by-side vehicles). The financial benefit of the rebate scheme was identified as a primary cue to action and key enabler.

Conclusion This study found that Victorian farmer quad bike users had a strong understanding of the dangers and risks involved in quad bike usage. Farmer perception of how these risks applied to their own setting was a major decision-making factor whether to take part in the rebate scheme.

Learning Outcomes The communication a farmer received was likely to influence their perception of rebate scheme benefits, as was the types of supports given. 